University of Nebraska - Lincoln

DigitalCommons@University of Nebraska - Lincoln

3-1989

\title{
Semi-Implicit and Fully Implicit Shock-Capturing Methods for Nonequilibrium Flows
}

Helen C. Yee

Judy L. Shinn

Follow this and additional works at: https://digitalcommons.unl.edu/nasapub

Part of the Astrophysics and Astronomy Commons

This Article is brought to you for free and open access by the National Aeronautics and Space Administration at DigitalCommons@University of Nebraska - Lincoln. It has been accepted for inclusion in NASA Publications by an authorized administrator of DigitalCommons@University of Nebraska - Lincoln. 


\title{
Semi-Implicit and Fully Implicit Shock-Capturing Methods for Nonequilibrium Flows
}

\author{
H. C. Yee* \\ NASA Ames Research Center, Moffett Field, California \\ and \\ Judy L. Shinn $\dagger$ \\ NASA Langley Research Center, Hampton, Virginia
}

\begin{abstract}
Some numerical aspects of finite-difference algorithms for nonlinear multidimensional hyperbolic conservation laws with stiff nonhomogeneous (source) terms are discussed. If the stiffness is entirely dominated by the source term, a semi-implicit shock-capturing method is proposed. However, if the stiffness is not solely dominated by the source terms, a fully implicit method would be a more efficient solution procedure. The primary motivation for constructing these schemes was to address large systems of thermally and chemically nonequilibrium flows in the hypersonic regime. Due to the unique structure of the eigenvalues and eigenvectors for fluid flows of this type, the computation can be simplified, thus providing a more efficient solution procedure than one might have anticipated. An implicit algorithm with explicit coupling between fluid and species equations is also proposed.
\end{abstract}

\section{Introduction}

T HE second-order total variation diminishing (TVD) type of schemes ${ }^{1-5}$ originally designed for nonlinear scalar hyperbolic conservation laws or constant coefficient hyperbolic systems has been proven to be applicable to many multidimensional fluid dynamics problems (especially for a perfect gas). See, for example, Refs. 6-11. In particular, the explicit and implicit TVD schemes originally developed by Harten, ${ }^{1}$ Roe, ${ }^{12}$ and Davis, ${ }^{13}$ and later modified and generalized by $\mathrm{Yee}^{2}$ are rather simple in structure and require the least computational effort among the class of TVD methods. For the two-dimensional, compressible, Euler equations for a perfect gas, these explicit TVD methods require approximately twice the CPU time as the classical Lax-Wendroff and MacCormack schemes. The gain in robustness and elimination of spurious oscillation by TVD schemes often justifies the extra computation. On the other hand, straightforward extension of these methods to include stiff source terms for large systems is prohibitively expensive in terms of efficiency. There has been little work in efficiently generalizing TVD methods to large systems with stiff source terms. In this paper, some numerical aspects and new procedures in treating nonequilibrium flows based on existing TVD properties will be discussed, including both semi-implicit and fully implicit methods.

Stiffness of Source Terms

Treating stiff problems implicitly is a common practice for the integration of stiff ordinary differential equations. ${ }^{14}$ To further speed up the solution procedure, Kreiss ${ }^{15}$ proposed

Received March 26, 1987; presented as Paper 87-1116 at the AIAA

8th Computational Fluid Dynamics Conference, Honolulu, HI, June

9-11, 1987; revision received Feb. 18, 1988. Copyright (C) 1988

American Institute of Aeronautics and Astronautics, Inc. No copy-

tight is asserted in the United States under Title 17, U.S. Code. The

the Government has a royalty-free license to exercise all rights under

the copyright claimed herein for Governmental purposes. All other

ghts are reserved by the copyright owner.

"Research Scientist, Computational Fluid Dynamics Branch.

Membospace Technologist, Aerothermodynamics Branch. Senior ember AIAA. splitting the equation into stiff and nonstiff terms and applying a special initial condition to damp out the fast wave initially. Guerra and Gustafsson ${ }^{16}$ extended these ideas by applying a semi-implicit method to time-dependent hyperbolic problems with varying time scales. Their technique is to treat the stiff part implicitly and at the same time damp out the fast wave, leaving a more manageable system. Their methodology works well for nearly incompressible flows or transonic flows. For general hypersonic applications, due to the existence of more than one stiff parameter, the situation is more complicated. However, if the stiffness is dominated by the source terms and steady-state solutions are especially desired, one can still use this idea.

In the area of both thermally and chemically nonequilibrium flows, the main terms responsible for the stiffness are the source terms. Kee and Miller ${ }^{17}$ used a first-order spatial differencing together with a fully implicit time-marching scheme in handling combustion-related problems. Bussing and Murman ${ }^{18}$ have illustrated that taking a second-order central difference in space and treating the source term implicitly will essentially rescale the equation in time so that all events occur on a similar pseudotime scale for steady-state chemical reaction calculations. Similarily, other researchers such as Refs. 11 and 19-23 have used some kind of implicit treatment for stiff problems.

\section{Classical vs Modern Shock-Capturing Schemes}

Most of the numerical methods for reacting flows ${ }^{17,18,20-23}$ just discussed used classical shock-capturing methods. They only exhibit accurate results for smooth or weak shock solutions and are not robust enough for hypersonic computations. This is typical of classical shock-capturing techniques that result in oscillatory solutions across the discontinuities and/or nonlinear instability, whereas the modern shock-capturing techniques such as the upwind and symmetric TVD schemes of Harten, ${ }^{1}$ Yee, ${ }^{2}$ Roe,${ }^{3,12}$ Osher and Chakravarthy, ${ }^{4}$ and Jameson and $\mathrm{Lax}^{5}$ do not possess these deficiencies. In particular, symmetric TVD schemes appear to be more attractive than upwind TVD schemes since symmetric schemes have a smaller operation count and are less sensitive to numerical boundary-condition treatments. ${ }^{7}$ An example is the current enhancement work to program LAURA by Gnoffo et 
al. ${ }^{11}$ that has resulted in a large savings in TVD operation steps as compared with the Osher and Chakravarthy method. ${ }^{4}$

Coupling or Uncoupling the Species Equation From the Fluid Equations

In the application of modern shock-capturing methods like the TVD type of schemes for the chemically reacting flows, Carofano ${ }^{10}$ was the first to introduce the formulation that enabled full coupling in Harten's explicit TVD scheme for a two-species, two-dimensional unsteady flow in Cartesian coordinates. However, due to the system size and the varying time-scale nature of the problem, the operations' count increases nonlinearly as the number of species increases. To avoid solving a large system, Gnoffo and McCandless ${ }^{19}$ and Gnoffo et al. ${ }^{11}$ uncoupled the species equations from the fluid dynamics equations and solved these two sets of systems of nonlinear partial-differential equations in a time-lag fashion (loosely coupled method) by using a point-relaxation technique with a second-order symmetric TVD scheme of $\mathrm{Yee}^{2}$ and an upwind TVD scheme of Osher and Chakravarthy. ${ }^{4}$ Eberhardt and Brown ${ }^{24}$ attempted to use the eigenvalues and eigenvectors of the fluid dynamics equations alone to obtain a "fully coupled" first-order explicit TVD scheme for a one-dimensional flow. The result of Eberhardt and Brown showed excessive smearing at the shock when compared with the true fully coupled explicit TVD result. Their motivation for designing such a coupling procedure was to optimize the operation count by avoiding multiplication of large matrices. However, as will be demonstrated in the present work, if one makes use of the unique structure of eigenvectors and eigenvalues for fluid flow of this type, the fully coupled formulation can be simplified even for a large number of species, thus providing a more efficient solution procedure than one might have anticipated. Moreover, using the eigenvalues and eigenvectors for the fully coupled equation set allows one to have the freedom of controlling the proper amount of numerical dissipation for the individual waves. ${ }^{7}$ In particular, for the two-dimensional chemically reacting flows, the number of linear waves is $n s+1$ in each spatial direction where $n s$ is the number of linearly independent species. Note that in order to capture contact discontinuities accurately, it is very important to apply the proper amount of numerical dissipation to the linear waves.

\section{Proposing New Schemes}

The goal of the present work is to construct second-order and third-order high-resolution schemes such that they are appropriate for large systems of thermally and chemically nonequilibrium flows in the hypersonic regime, and, at the same time, they are efficient and simple to program and easy to implement into existing computer codes. The approach here is to improve the existing technology by combining the desirable features from a few existing numerical methods into a single algorithm and, most important, by taking into account the specific characteristics of the particular physical problem.

Two types of schemes are proposed for steady-state computations. If the stiffness is entirely dominated by the source term, a semi-implicit TVD type of shock-capturing method is proposed. However, if the stiffness is not solely dominated by the source terms (e.g., stiffness due to highly irregular grid and/or viscous flows), a fully implicit method would be a more efficient procedure. The proposed fully implicit relaxation algorithm can be viewed as a variant of a fully coupled form of the algorithm proposed by Gnoffo and McCandless. ${ }^{19}$ An implicit algorithm with explicit coupling between fluid and species equations proposed by $\mathrm{Yee}^{25,26}$ will also be included here. Many existing perfect gas or equilibrium real-gas computer codes can be modified easily to include this algorithm, which is a compromise between the loosely coupled implicit method of Ref. 19 and the fully coupled, fully implicit TVD method proposed here. The schemes discussed here can, in principle, be used for unsteady nonequilibrium flows. How. ever, new difficulties arise when designing shock-capturing methods for unsteady nonequilibrium flows that do not arise for their steady-state counterpart. A study of these aspects is reported in LeVeque and Yee. ${ }^{27}$

Due to space limitations, the material is divided into two separate papers. The mathematical aspects and derivation of the numerical methods for solving the two-dimensional hypersonic nonequilibrium flow in Cartesian coordinates are given in this paper, whereas the extension and application of the semi-implicit method to the three-dimensional, fully coupled chemically reacting viscous flow in generalized coordinates are presented in a companion paper. ${ }^{28}$ Numerical experiments for the fully implicit scheme are reported in a paper by Gnoffo. ${ }^{29}$ In the following sections, the proposed numerical methods for both the semi-implicit and fully implicit shock-capturing schemes will be described briefly. A test example will also be included to illustrate the performance of the proposed semi-implicit scheme.

\section{Basic Explicit Predictor-Corrector Scheme}

In this section, the predictor-corrector form of the basic explicit TVD scheme is given. Also, all the necessary terms that are required for the basic TVD scheme for the compressible inviscid chemically reacting flow equations are derived.

\section{Governing Equations}

Consider a two-dimensional system of nonhomogeneous hyperbolic conservation laws

$$
\frac{\partial U}{\partial t}+\frac{\partial F(U)}{\partial x}+\frac{\partial G(U)}{\partial y}=S(U)
$$

Here, $\boldsymbol{U}, \boldsymbol{F}(\boldsymbol{U}), \boldsymbol{G}(\boldsymbol{U})$, and $\boldsymbol{S}(\boldsymbol{U})$ are column vectors of $k$ components. Let $\boldsymbol{A}=\partial \boldsymbol{F} / \partial \boldsymbol{U}$ and $\boldsymbol{B}=\partial \boldsymbol{G} / \partial \boldsymbol{U}$, with $\left(a_{x}^{1}, a_{x}^{2}, \ldots, a_{x}^{k}\right)$ and $\left(a_{y}^{1}, a_{y}^{2}, \ldots, a_{y}^{k}\right)$ being the eigenvalues of $\boldsymbol{A}$ and $\boldsymbol{B}$, respectively. Denote $\boldsymbol{R}_{x}$ and $\boldsymbol{R}_{y}$ as the matrices whose columns are eigenvectors of $\boldsymbol{A}$ and $\boldsymbol{B}$ and denote $\boldsymbol{R}_{x}^{-1}$ and $\boldsymbol{R}_{y}^{-1}$ as the inverses of $\boldsymbol{R}_{x}$ and $\boldsymbol{R}_{y}$. In the case of the compressible inviscid flow equations with chemical reactions, when the global continuity equation is replaced by the individual species continuity equations,

$$
\begin{gathered}
U=\left(\rho^{1}, \ldots, \rho^{n s}, m, n, e\right)^{T} \\
\boldsymbol{F}(\boldsymbol{U})=\left[c^{1} m, \ldots, c^{n s} m, \frac{m^{2}}{\rho}+p, \frac{m n}{\rho},(e+p) \frac{m}{\rho}\right]^{T} \\
\boldsymbol{G}(\boldsymbol{U})=\left[c^{1} n, \ldots, c^{n s} n, \frac{m n}{\rho}, \frac{n^{2}}{\rho}+p,(e+p) \frac{n}{\rho}\right]^{T} \\
S(\boldsymbol{U})=\left(s^{1}, \ldots, s^{n s}, 0,0,0\right)^{T}
\end{gathered}
$$

Note that the current formulation and the particular property of the schemes (to be discussed) remain if $n s-1$ species and the global continuity equations were used. Here, $m=\rho u$, $n=\rho v$, and $s^{i}$ represents the production of species from chemical reactions. The variables are the velocity components $u$ and $v$, the pressure $p$, the total energy per unit volume $e$, and the density of the $i$ th species $\rho^{i}$. Also, $\rho=\sum_{i=1}^{n s} \rho^{i}$ and $c^{i} \rho=\rho^{i}$, where $n s$ is the number of species in the model and $c^{i}$ is the species mass fraction. Equations (2) assume that all the $\rho^{i}$ are linearly independent.

The eigenvalues of $\boldsymbol{A}$ and $\boldsymbol{B}$ are

$$
\begin{aligned}
& \left(a_{x}^{1}, \ldots, a_{x}^{n s+3}\right)=(u, \ldots, u, u+a, u, u-a) \\
& \left(a_{y}^{1}, \ldots, a_{y}^{n s+3}\right)=(v, \ldots, v, v+a, v, v-a)
\end{aligned}
$$


Here, the so-called "frozen sound speed" $a$ is

$$
a^{2}=p_{\rho}+p_{e}\left(H-u^{2}-v^{2}\right)
$$

with

$$
\begin{aligned}
p_{\rho} & =\sum_{i=1}^{n s} c^{i} p_{\rho^{i}} \\
\sum_{i=1}^{n s} c^{i} & =1 \\
p_{\rho^{i}} & =\left.\frac{\partial p}{\partial \rho^{i}}\right|_{m, n, e, \ldots} \\
p_{e} & =\left.\frac{\partial p}{\partial e}\right|_{m, n, \rho^{1}, \ldots} \\
H & =\frac{e+p}{\rho}
\end{aligned}
$$

Let the grid spacing be denoted by $\Delta x$ and $\Delta y$ such that $x=j \Delta x$ and $y=k \Delta y$, and let the time-step be denoted by $\Delta t$ such that $t=n \Delta t$ where the superscript $n$ is used for the time index and should not be confused with the $n=\rho u$ in Eq. (2). Let $a_{j+1 / 2}^{l}, \boldsymbol{R}_{j+1 / 2}, \boldsymbol{R}_{j+1 / 2}^{-1 / 2}$ denote the quantities $a_{x}^{l}, \boldsymbol{R}_{x}, \boldsymbol{R}_{x}^{-1}$ evaluated at some symmetric average of $\boldsymbol{U}_{j, k}$, and $\boldsymbol{U}_{j+1, k}$. Similarly, let $a_{k+1 / 2}, \boldsymbol{R}_{k+1 / 2}, \boldsymbol{R}_{k+1 / 2}^{-1 / 2}$ denote the quantities $a_{y}^{l}$, $\boldsymbol{R}_{y}, \boldsymbol{R}_{y}^{-1}$ evaluated at some symmetric average of $\boldsymbol{U}_{j, k}$ and $U_{j k+1}$. In the case of the chemically reacting flows, $a_{j+1 / 2}^{l}$, $\boldsymbol{R}_{j+1 / 2}, \boldsymbol{R}_{j+1 / 2}^{-1 / 2}, a_{k+1 / 2}, \boldsymbol{R}_{k+1 / 2}$, and $\boldsymbol{R}_{k+1 / 2}^{-1 / 2}$ are defined (with modification) in ways similar to those used by Huang, ${ }^{30}$ Carofano, ${ }^{10}$ and Roe. ${ }^{31}$ See a review paper by $\mathrm{Yee}^{26}$ for details. Define

$$
\boldsymbol{\alpha}_{j+1 / 2}=\boldsymbol{R}_{j+1 / 2}^{-1 / 2}\left(\boldsymbol{U}_{j+1, k}-\boldsymbol{U}_{j, k}\right)
$$

as the difference (or the jump) of the characteristic variables in the locally $x$ direction and define

$$
\boldsymbol{\alpha}_{k+1 / 2}=\boldsymbol{R}_{k+1 / 2}^{-1 / 2}\left(\boldsymbol{U}_{j, k+1}-\boldsymbol{U}_{j, k}\right)
$$

as the difference of the characteristic variables in the locally $y$ direction. For thermally and chemically nonequilibrium flows, the eigenvalues and eigenvectors have a similar structure. For the two-dimensional system (1), if $n t$ is the number of thermal energy variables, then the eigenvalues in the $x$ direction will have $(n s+n t+1) u$ characteristics plus $u+a$ and $u-a$ characteristics. Here, the values $a$ will reflect the added thermal energy variables. ${ }^{48}$

\section{Explicit Predictor-Corrector TVD Scheme}

With the foregoing notation, a formal extension of the scalar explicit second-order TVD method ${ }^{2,26}$ in predictorcorrector form via the local characteristic approach for the nonlinear hyperbolic system, Eq. (1), can be written as

$$
\Delta U_{j, k}^{(1)}=-\frac{\Delta t}{\Delta x}\left(F_{j, k}^{n}-F_{j-1, k}^{n}\right)-\frac{\Delta t}{\Delta y}\left(G_{j, k+1}^{n}-G_{j, k}^{n}\right)+\Delta t S_{j, k}^{n}
$$

$$
\boldsymbol{U}_{j, k}^{(1)}=\Delta \boldsymbol{U}_{j, k}^{(1)}+\boldsymbol{U}_{j, k}^{n}
$$

$$
\begin{aligned}
& \Delta U_{j, k}^{(2)}=\frac{1}{2}\left\{-\Delta U_{j, k}^{(1)}-\frac{\Delta t}{\Delta x}\left(F_{j+1, k}^{(1)}-F_{j, k}^{(1)}\right)\right. \\
& \left.-\frac{\Delta t}{\Delta y}\left(G_{j, k}^{(1)}-G_{j, k-1}^{(1)}\right)+\Delta t S_{j, k}^{(1)}\right\}
\end{aligned}
$$

$$
\boldsymbol{U}_{j, k}^{(2)}=\Delta \boldsymbol{U}_{j, k}^{(2)}+\boldsymbol{U}_{j, k}^{(1)}
$$

$$
\begin{aligned}
& \boldsymbol{U}_{j, k}^{n+1}=\boldsymbol{U}_{j, k}^{(2)}+\left(\boldsymbol{R}_{j+1 / 2}^{(2)} \boldsymbol{\Phi}_{j+1 / 2}^{(2)}-\boldsymbol{R}_{j-1 / 2}^{(2)} \boldsymbol{\Phi}_{j-1 / 2}^{(2)}\right) \\
& \quad+\left(\boldsymbol{R}_{k+1 / 2}^{(2)} \boldsymbol{\Phi}_{k+1 / 2}^{(2)}-\boldsymbol{R}_{k-1 / 2}^{(2)} \boldsymbol{\Phi}_{k-1 / 2}^{(2)}\right)
\end{aligned}
$$

A detailed discussion of Eqs. (12) can be found in Yee. ${ }^{26} \mathrm{Here}$, the superscripts (1) and (2) designate the values of the function evaluated at the intermediate solutions $\boldsymbol{U}^{(1)}$ and $\boldsymbol{U}^{(2)}$. The elements of the vector $\boldsymbol{\Phi}_{j+1 / 2}$, denoted by $\left(\phi_{j+1 / 2}^{l}\right)^{S}$ with $l=1, \ldots, k$ for a symmetric TVD-type scheme, are

$$
\begin{gathered}
\left(\phi_{j+1 / 2}^{l}\right)^{S}=1 / 2\left[\psi\left(v_{j+1 / 2}^{l}\right)-\left(\nu_{j+1 / 2}^{l}\right)^{2}\right]\left(\alpha_{j+1 / 2}^{l}-\hat{Q}_{j+1 / 2}^{l}\right) \\
\nu_{j+1 / 2}^{l}=\frac{\Delta t}{\Delta x} a_{j+1 / 2}^{l}
\end{gathered}
$$

where $\alpha_{j+1 / 2}^{l}$ are elements of Eq. (10) and $a_{j+1 / 2}^{l}$ are eigenvalues in the $x$ direction. The function $\psi$ is

$$
\psi(z)= \begin{cases}|z| & |z| \geq \epsilon \\ \left(z^{2}+\epsilon^{2}\right) / 2 \epsilon & |z|<\epsilon\end{cases}
$$

Here, $\psi(z)$ in Eq. (13c) is an entropy correction to $|z|$ where $\epsilon$ is a small positive parameter. The parameter $\epsilon$ can be a function of $a_{x}^{l}$ and $a_{y}^{l}$. In particular, for multidimensional hypersonic steady-state computations, the choice of $\epsilon$ is very important, and it is highly Mach-number- and geometry-dependent on the physical problem. See Refs. 26, 32, and 33 for a discussion. Examples of the "limiter" function $\hat{Q}_{j+1 / 2}^{l}$ can be expressed as

$$
\begin{aligned}
\hat{Q}_{j+1 / 2}^{l} & =\operatorname{minmod}\left(\alpha_{j-1 / 2}^{l}, \alpha_{j+1 / 2}^{l}\right) \\
\quad+\operatorname{minmod}\left(\alpha_{j+1 / 2}^{l}, \alpha_{j+3 / 2}^{l}\right)-\alpha_{j+1 / 2}^{l} & \\
\hat{Q}_{j+1 / 2}^{\prime} & =\operatorname{minmod}\left(\alpha_{j-1 / 2}^{l}, \alpha_{j+1 / 2}^{l}, \alpha_{j+3 / 2}^{l}\right) \\
\hat{Q}_{j+1 / 2}^{l} & =\operatorname{minmod}\left[2 \alpha_{j-1 / 2}^{l}, 2 \alpha_{j+1 / 2}^{l}, 2 \alpha_{j+3 / 2}^{l}, 1 / 2\left(\alpha_{j-1 / 2}^{l}+\alpha_{j+3 / 2}^{l}\right)\right]
\end{aligned}
$$

The minmod function of a list of arguments is equal to the smallest number in absolute value if the list of arguments is of the same sign or is equal to zero if any arguments are of opposite sign. Due to the nonuniqueness of the eigenvectors, the choice of Eqs. (10) and (11) on which the limiters are applied plays an important role in the convergence rate as the Mach number increases. See Refs. 32 and 33 for details.

The formulation of this scheme is purposely broken into two parts, namely, the predictor-corrector step of the MacCormack explicit scheme and an appropriate conservative dissipation term designed in such a way that the final scheme is TVD for the one-dimensional constant coefficient case. It is a slight modification of Roe's one-step TVD Lax-Wendroff scheme. If one sets $\hat{Q}$ to be Eq. (13d) and $\psi(z)=|z|$, the scalar scheme [with $S=0$ and with the second and third terms in Eq. (12e) evaluated at $U^{n}$ ] is the same as described in Davis $^{13}$ and Kwong. ${ }^{34}$ The reason for choosing the predictorcorrector step instead of the one-step Lax-Wendroff formulation is that the predictor-corrector form provides a natural and efficient inclusion of the source terms for multidimensional problems. ${ }^{26,35}$ The second-order scheme, [Eq. (12)] belongs to the class of symmetric TVD-type methods as discussed in Ref. 2. By defining a more complex $\phi_{j+1 / 2}^{l}$, the scheme of Eq. (12) can be made upwind-weighted and would belong to the class of upwind TVD-type schemes.

Another numerical aspect is that the method of extending scalar TVD schemes to nonlinear systems of hyperbolic conservation laws (1) in Eq. (12) by using the eigenvalues and eigenvectors of $\partial F / \partial U$ and $\partial G / \partial U$ is sometimes referred to as 
the local characteristic approach and is a variant of Roe's approximate Riemann solver. ${ }^{31}$ It is more efficient than the exact or other approximate Riemann solvers, and it provides a natural way to linearize the implicit TVD schemes. ${ }^{8,36}$ The current approach, in effect, uses scalar schemes on each characteristic field so that the limiter used need not be the same for each field. One can even use different schemes for different fields. For the hyperbolic system, Eqs. (1-9), the characteristic fields consist of two nonlinear fields and $(n s+1)$ linear fields of the same value in each direction. The contact discontinuities are associated with the linear fields. It has been shown ${ }^{2,7,9,26}$ that the two different fields required different amounts of numerical dissipation (i.e., different limiters). Often, the limiters that are designed for the linear field might give spurious oscillation or nonphysical solutions for the nonlinear field.

\section{More Efficient Solution Procedures}

The extra computation in Eq. (12) compared with the classical central difference shock-capturing scheme such as the Lax-Wendroff method is due to the vectors $(\boldsymbol{R} \Phi)_{j \pm 1 / 2}$. At first glance, the vectors $\alpha_{j \pm 1 / 2}$ and $(\boldsymbol{R} \Phi)_{j \pm 1 / 2}$ involve matrix and vector multiplication of dimension $n s+3$ for every grid point and thus tend to discourage their adaption to problems other than ideal gas flows. Researchers such as Gnoffo and McCandless ${ }^{19}$ and Eberhardt and Brown ${ }^{24}$ were motivated to pursue other avenues to solve the complicated chemically reacting flow problems. However, if one makes use of the unique structure of the eigenvectors and eigenvalues for fluid flow of this type, the fully coupled formulation can be simplified even for a large number of species and thus becomes a viable approach.

With straightforward manipulations, the operation for the scheme of Eq. (12) can be simplified tremendously. The vector $\alpha$ in Eq. (10), for example, can be expressed as

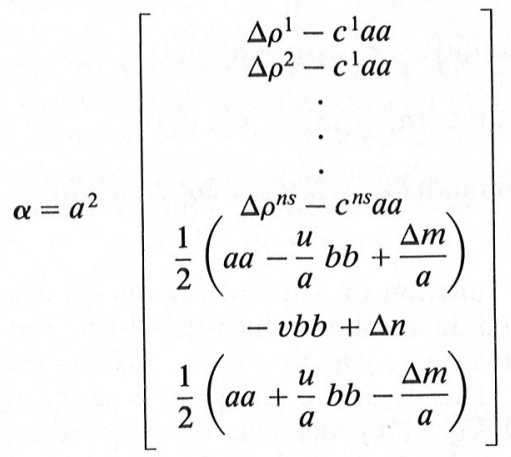

with

$$
\begin{gathered}
a a=\frac{1}{a^{2}}\left[\sum_{i=1}^{n s} p_{\rho^{i}} \Delta \rho^{i}-p_{e}(u \Delta m+v \Delta n-\Delta e)\right] \\
b b=\sum_{i=1}^{n s} \Delta \rho^{i}
\end{gathered}
$$

Here, for example, $\left(\Delta \rho^{i}\right)_{j+1 / 2}=\rho_{j+1, k}^{i}-\rho_{j, k}^{i}$ and it is understood that the spatial indices in Eqs. $(14-16)$ are at $(j+1 / 2, k)$. Similarly, $\boldsymbol{R} \Phi$ also has a very simple form. For instance, the $\boldsymbol{R} \Phi$ associated with the $x$-direction flux can be expressed as

$$
R \Phi=\frac{1}{a^{2}}\left[\begin{array}{c}
\phi^{1}+c^{1} k_{1} \\
\phi^{2}+c^{2} k_{1} \\
\cdot \\
\cdot \\
\phi^{n s}+c^{n s} k_{1} \\
u k_{2}+k_{3} \\
v k_{2}+\phi^{n s+2} \\
H k_{2}-\frac{1}{p_{e}} \sum_{i=1}^{n s}\left(a^{i}\right)^{2} \phi^{i}+v \phi^{n s+2}+u k_{3}
\end{array}\right]
$$

with

$$
\begin{aligned}
& k_{1}=\phi^{n s+1}+\phi^{n s+3} \\
& k_{2}=\sum_{i=1, i \neq n s+2}^{n s+3} \phi^{i} \\
& k_{3}=a\left(\phi^{n s+1}-\phi^{n s+3}\right)
\end{aligned}
$$

and $\left(a^{i}\right)^{2}$ is the square of the frozen sound speed for the $i$ th species. Here, the spatial indices on Eq. (17) are at $(j+1 / 2, k)$. As one can see, the terms in Eqs. (14) and (17) due to the species equations require only two operations per species, Therefore, the increase in the number of species equations is not as "CPU" intensive as one might have anticipated.

\section{Semi-Implicit Predictor-Corrector TVD Scheme}

The explicit TVD scheme of Eq. (12) can be used for either time-accurate or steady-state calculation. However, if the source term is stiff, the restriction in the time-step due to the stability requirement is prohibitively small and Eq. (12) is not practical, especially for steady-state applications. In this section, a semi-implicit method is proposed. Another alternative is a fully implicit method. The basic implicit scheme and a simplified procedure will be discussed in Sec. IV.

If one follows the idea of Bussing and Murman ${ }^{18}$ in treating the source term implicitly, a semi-implicit predictor-corrector TVD-type scheme can be easily obtained. It can be written as a one-parameter family of time-differencing schemes for the source term; i.e., the following formulation includes the scheme of Eq. (12). The proposed scheme can be written as

$$
\begin{aligned}
& D_{j, k}^{n} \Delta U_{j, k}^{(1)}=-\frac{\Delta t}{\Delta x}\left(F_{j, k}^{n}-F_{j-1, k}^{n}\right) \\
& -\frac{\Delta t}{\Delta y}\left(G_{j, k+1}^{n}-G_{j, k}^{n}\right)+\Delta t S_{j, k}^{n} \\
& D=\left(I-\Delta t \theta \frac{\partial S}{\partial U}\right) \\
& \boldsymbol{D}_{j, k}^{(1)} \Delta \boldsymbol{U}_{j, k}^{(2)}=\frac{1}{2}\left\{\Delta \boldsymbol{U}_{j, k}^{(1)}-\frac{\Delta t}{\Delta x}\left(\boldsymbol{F}_{j+1, k}^{(1)}-\boldsymbol{F}_{j, k}^{(1)}\right)\right. \\
& \left.-\frac{\Delta t}{\Delta y}\left(G_{j, k}^{(1)}-G_{j, k-1}^{(1)}\right)+\Delta t S_{j, k}^{(1)}\right\} \\
& \boldsymbol{U}_{j, k}^{n+1}= \\
& \boldsymbol{U}_{j, k}^{(2)}+\left[\boldsymbol{R}_{j+1 / 2}^{(2)} \boldsymbol{\Phi}_{j+1 / 2}^{(2)}-\boldsymbol{R}_{j-1 / 2}^{(2)} \boldsymbol{\Phi}_{j-1 / 2}^{(2)}\right] \\
& +\left[\boldsymbol{R}_{k+1 / 2}^{(2)} \boldsymbol{\Phi}_{k+1 / 2}^{(2)}-\boldsymbol{R}_{k-1 / 2}^{(2)} \boldsymbol{\Phi}_{k-1 / 2}^{(2)}\right]
\end{aligned}
$$

The order of accuracy for Eqs. (18) is highly dependent on how one evaluates $S^{(1)}$ in Eq. (18c) and the second and third terms in Eq. (18d). As it stands, Eq. (18) is first-order in time. The scheme can be made second-order in time if one evaluates these terms at $U^{(n)}$ instead of $U^{(1)}$ and $U^{(2)}$. See Ref. 27 for details. Here, $\boldsymbol{D}$ is assumed to be invertible; i.e., only the type of source terms with its Jacobian such that $\boldsymbol{D}$ is invertible at each grid point is permissible. The parameter $\theta$ is in the range $0 \leq \theta \leq 1$. For $\theta \neq 0$, the source terms are treated implicitly. $\theta=1$, the time-differencing for the source term is first-orde and Eqs. (18) are best suited for steady-state calculations. Note that the second corrector step, Eq. (18d), is the important part of the algorithm that deviates from the Bussing and Murman method. This step, which ensures that method will have the TVD-type properties, is designed capture shock waves without the associated spurious oscil tions. 
One can simplify Eqs. (18) by partitioning the vectors $U, F, G, S, D$ in Eq. (2) as follows:

$$
\begin{gathered}
U=\left[\begin{array}{c}
\boldsymbol{U}^{I} \\
\boldsymbol{U}^{I I}
\end{array}\right], \quad \boldsymbol{U}^{I}=\left[\begin{array}{c}
u^{1} \\
\vdots \\
\cdot \\
u^{n s}
\end{array}\right], \quad \boldsymbol{U}^{I I}=\left[\begin{array}{l}
u^{n s+1} \\
u^{n s+2} \\
u^{n s+3}
\end{array}\right] \\
\boldsymbol{F}=\left[\begin{array}{c}
\boldsymbol{F}^{I} \\
\boldsymbol{F}^{I I}
\end{array}\right], \quad \boldsymbol{G}=\left[\begin{array}{c}
\boldsymbol{G}^{I} \\
\boldsymbol{G}^{I I}
\end{array}\right] \\
S=\left[\begin{array}{c}
\boldsymbol{S}^{I} \\
\boldsymbol{S}^{I I}
\end{array}\right], \quad S^{I}=\left[\begin{array}{c}
s^{1} \\
\vdots \\
s^{n s}
\end{array}\right], \quad S^{I I}=\left[\begin{array}{l}
0 \\
0 \\
0
\end{array}\right] \\
D=\left[\begin{array}{ll}
D^{11} & D^{12} \\
D^{21} & D^{22}
\end{array}\right]
\end{gathered}
$$

Here, $D^{21}$ is a null matrix and $D^{22}$ is an identity matrix. With the aforementioned definitions, the scheme can be greatly simplified. The procedures are as follows: Taking the predictor step, for example, one first solves for $\left(\Delta U^{I I}\right)^{(1)}$ by

$$
\begin{gathered}
\left(\Delta U^{I I}\right)_{j, k}^{(1)}=-\frac{\Delta t}{\Delta x}\left[\left(F_{j, k}^{I I}\right)^{n}-\left(F_{j-1, k}^{I I}\right)^{n}\right] \\
-\frac{\Delta t}{\Delta y}\left[\left(G_{j, k+1}^{I I}\right)^{n}-\left(G_{j, k}^{I I}\right)^{n}\right]
\end{gathered}
$$

then for $\left(\Delta U^{I}\right)^{(1)}$ by

$$
\left(D_{j, k}^{11}\right)^{n}\left(\Delta U^{I}\right)_{j, k}^{(1)}=(\mathrm{rhs})^{I}-\boldsymbol{D}_{j, k}^{12}\left(\Delta \boldsymbol{U}^{I I}\right)_{j, k}^{(1)}
$$

where $(\mathrm{rhs})^{I}$ is the right-hand side of Eq. (20a) with all the indices II replaced by $I$ and with the term $\Delta t\left(S_{j, k}^{I}\right)^{n}$ added. In other words, one only has to invert the $\boldsymbol{D}^{11}$ matrix of dimension $(n s, n s)$ instead of $(n s+3, n s+3)$. Similarly, one can simplify the corrector step in the same way. The solution obtained from the above procedure is used to solve Eq. (18d).

In the case where $S^{I I}$ is not a null vector and it is not stiff, Eqs. (20) are still applicable, except one has to add the term $\Delta t\left(S_{j, k}^{I L}\right)^{n}$ to the right-hand side of Eq. (20a). In the steady-state calculations where body-fitted coordinates are used, one can further speed up the convergence rate by using a local timestepping approach..$^{37,38}$

To verify the current approach and to make a fair comparison with a known method, the proposed semi-implicit scheme was implemented into an existing three-dimensional code. ${ }^{23}$ This existing full Navier-Stokes code, originally developed by A. Kumar at NASA Langley Research Center, contains the explicit MacCormack scheme with source terms treated implicitly. A detailed description of a numerical experiment and the extension of Eqs. (18) to three-dimensional, chemically reacting flows in generalized coordinates is given in a companion paper. ${ }^{28}$ Only partial results from Ref. 28 are presented here to illustrate the performance of the proposed semi-implicit scheme.

Figures 1-3 show a preliminary test result for a three-dimensional, five-species viscous reacting flow using the semi-imclicit TVD scheme, Eqs. (18), together with Eq. (13f), as compared with an existing classical shock-capturing method that supplies numerical dissipation linearly. ${ }^{23}$ The numerical oscill for the semi-implicit TVD method is shown to be oscillation-free around the shock, whereas the time spent per
iterlation-forits iteration is approximately double when compared with the

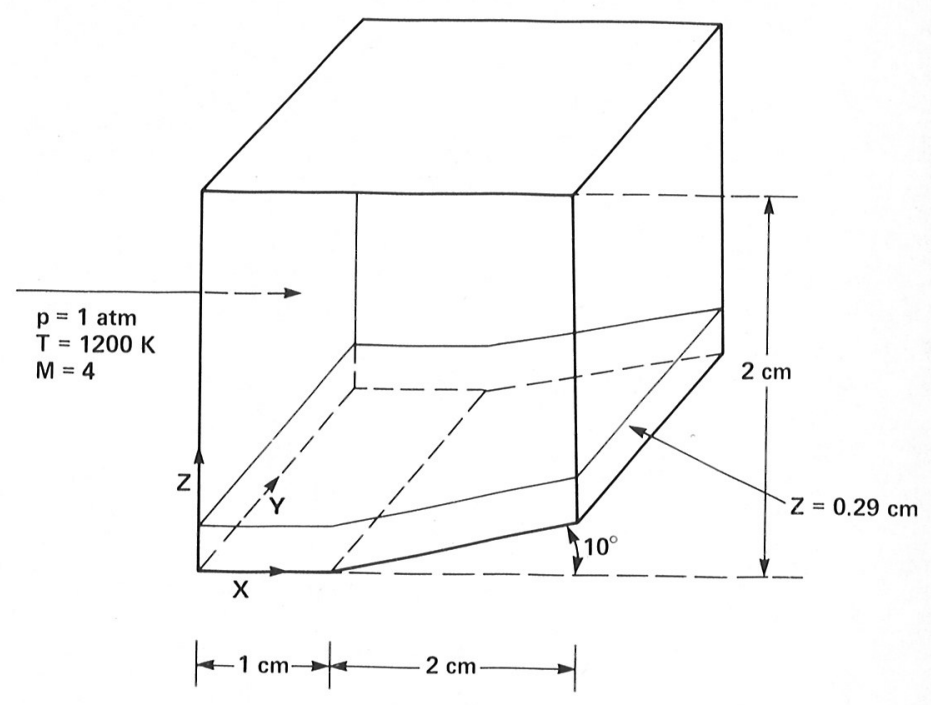

Fig. 1 Geometry and inflow condition.
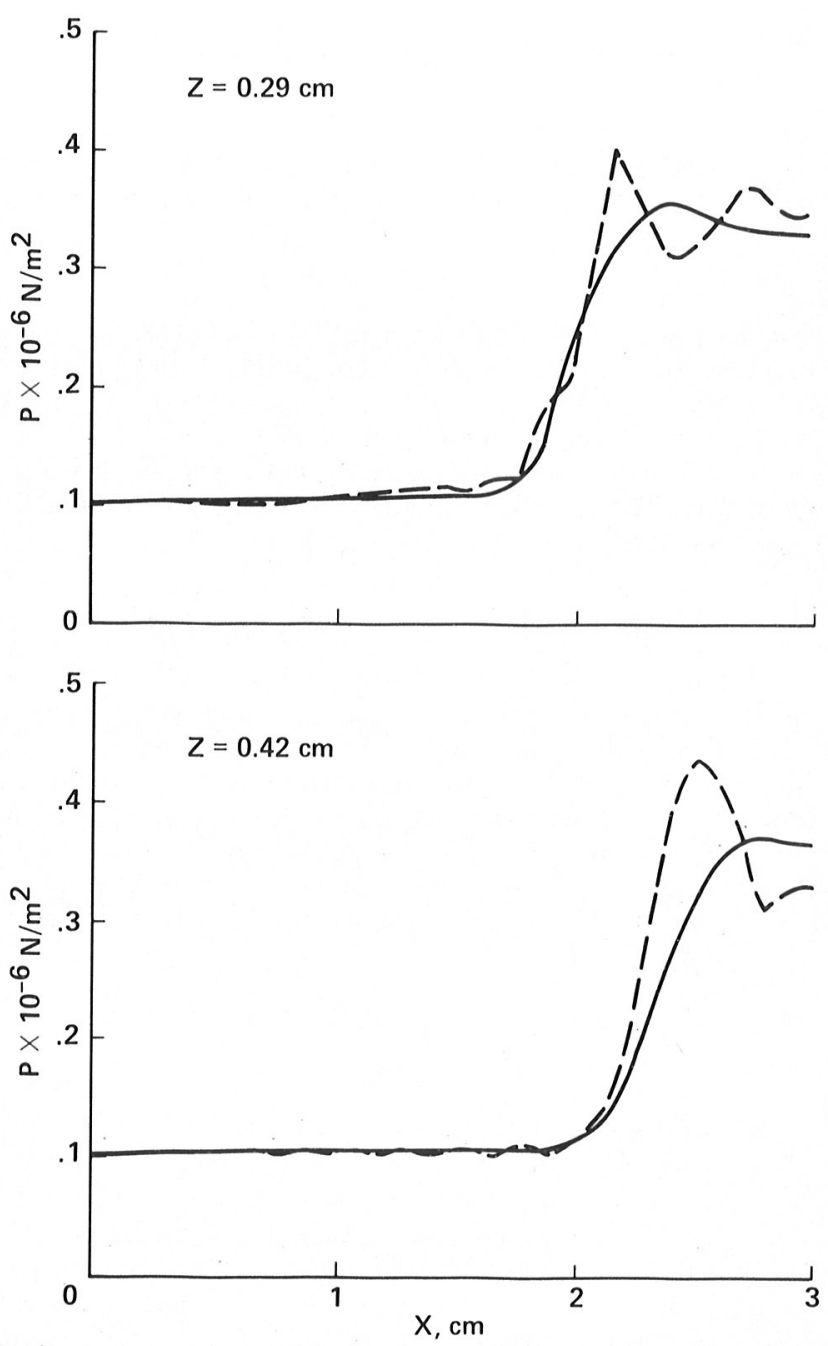

Fig. 2 Pressure profiles along $z=0.29$ and $z=0.42 \mathrm{~cm}$; - semiimplicit TVD method; -- classical shock-capturing method.

method used in Ref. 23. The configuration of the numerical experiment is shown in Fig. 1. Although this is a two-dimensional flow, to check out the three-dimensional code, it is computed as a three-dimensional flow with the appropriate boundary conditions in the $y$ direction. A uniform grid consisting of 31 points in the $x$ direction, 6 points in the $y$ 


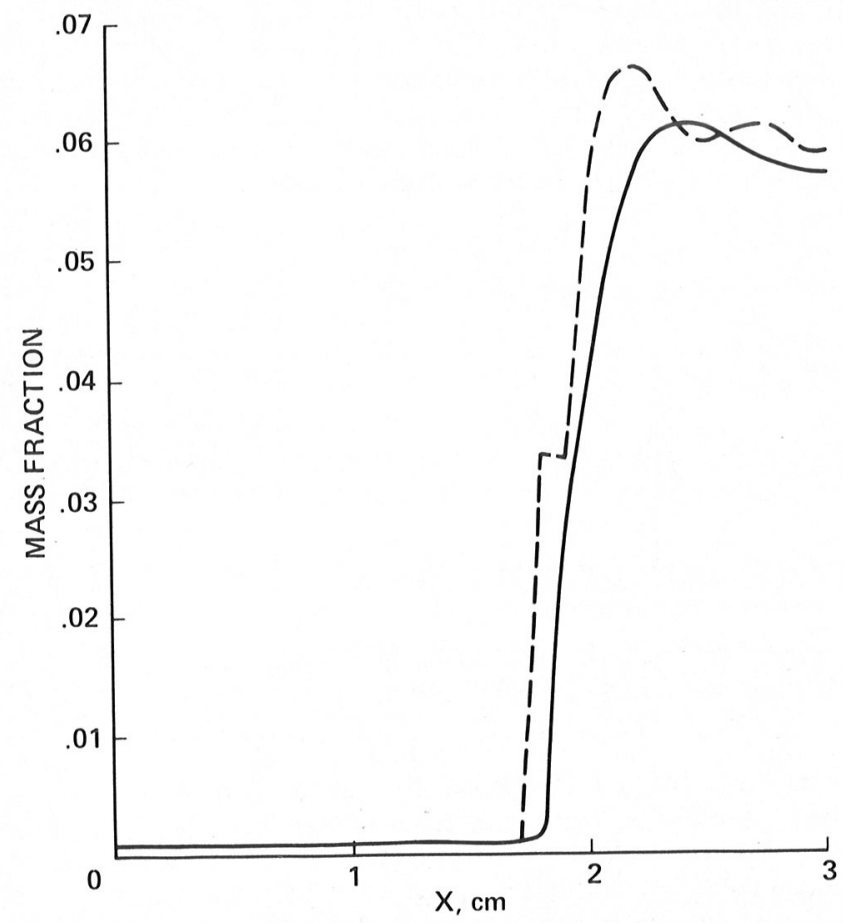

Fig. 3 Species distribution for $O H$ along $z=0.29 \mathrm{~cm}$; - semiimplicit TVD method; -- classical shock-capturing method.

direction, and a viscous grid consisting of 51 points in the $z$ direction were used. The inflow conditions are the pressure $p=1 \mathrm{~atm}$, the temperature $T=1200 \mathrm{~K}$, and the Mach number $M=4$ for a premixed air and hydrogen fuel. The species considered are $\mathrm{H}_{2}, \mathrm{O}_{2}, \mathrm{OH}, \mathrm{H}_{2} \mathrm{O}$, and $\mathrm{N}_{2}$, with $\mathrm{N}_{2}$ being inert. The so-called "global two-step chemistry model", for combustion ${ }^{23}$ is used.

\section{Fully Implicit TVD Method}

Another type of shock-capturing schemes with which the authors are familiar is one-parameter and two-parameter families of explicit and implicit TVD-type of schemes. ${ }^{2,26,33,36}$ The two-parameter family that includes the second-order implicit time-differencing that is suitable for unsteady viscous and inviscid flows as well as steady viscous computations is described in Yee et al. ${ }^{33}$ For steady-state inviscid nonequilibrium flows, the one-parameter family is sufficient and can be written as

$$
\begin{aligned}
& \boldsymbol{U}_{j, k}^{n+1}+\theta\left[\frac{\Delta t}{\Delta x}\left(\tilde{\boldsymbol{F}}_{j+1 / 2, k}^{n+1}-\tilde{\boldsymbol{F}}_{j-1 / 2, k}^{n+1}\right)\right. \\
& \left.\left.+\frac{\Delta t}{\Delta y}\left(\tilde{\boldsymbol{G}}_{j, k+1 / 2}^{n+1}-\tilde{\boldsymbol{G}}_{j, k}^{n+1}\right)-\Delta t / 2\right) \boldsymbol{S}_{j, k}^{n+1}\right] \\
& =\boldsymbol{U}_{j, k}^{n}-(1-\theta)\left[\frac{\Delta t}{\Delta x}\left(\tilde{\boldsymbol{F}}_{j+1 / 2, k}^{n}-\tilde{\boldsymbol{F}}_{j-1 / 2, k}^{n}\right)\right. \\
& \left.+\frac{\Delta t}{\Delta y}\left(\tilde{\boldsymbol{G}}_{j, k+1 / 2}^{n}-\tilde{\boldsymbol{G}}_{j, k-1 / 2}^{n}\right)-\Delta t \boldsymbol{S}_{j, k}^{n}\right]
\end{aligned}
$$

For $\theta \neq 0$, the scheme is now fully implicit. The $\tilde{\boldsymbol{F}}_{j \pm 1 / 2, k}$ and $\widetilde{\boldsymbol{G}}_{j, k \pm 1 / 2}$ are sometimes referred to as numerical flux functions. The spatial accuracy of the scheme depends on the form of the numerical flux functions. There exists many ways to achieve higher-order spatial accuracy and at the same time have TVD-type properties. Here, two ways are discussed. The first is due to Harten, ${ }^{1}$ Roe, ${ }^{3}$ and Yee, ${ }^{2}$ and the second, sometimes referred to as the MUSCL approach, is due to van Leer. ${ }^{39}$
Hereafter, we refer to the first way as the non-MUSCI approach.

\section{Non-MUSCL Approach}

The numerical flux function $\tilde{\boldsymbol{F}}_{j+1 / 2, k}$ for a non-MUSCL-type approach, together with Roe's type of approximate Riemann solver, for both the upwind and symmetric TVD schemes 2,8 can be expressed as

$$
\tilde{\boldsymbol{F}}_{j+1 / 2, k}=1 / 2\left(\boldsymbol{F}_{j, k}+\boldsymbol{F}_{j+1, k}+\boldsymbol{R}_{j+1 / 2} \boldsymbol{\Phi}_{j+1 / 2}\right)
$$

Similarly, one can define the numerical flux $\widetilde{\boldsymbol{G}}_{j, k+1 / 2}$ in this manner. The elements of the $\Phi_{j+1 / 2}$ denoted by $\left(\phi_{j+1 / 2}^{l}\right)^{S}$ for a general second-order symmetric TVD scheme are

$$
\left(\phi_{j+1 / 2}^{l}\right)^{S}=-\psi\left(a_{j+1 / 2}^{l}\right)\left(\alpha_{j+1 / 2}^{l}-\hat{Q}_{j+1 / 2}^{l}\right)
$$

where $\alpha_{j+1 / 2}^{\prime}$ are elements of Eq. (10). The function $\psi$ is Eq. (13c). The limiter function $\hat{Q}_{j+1 / 2}^{\prime}$ can be the same as that in Eqs. (13d-13f).

The elements of the $\boldsymbol{\Phi}_{j+1 / 2}$ denoted by $\left(\phi_{j+1 / 2}^{l}\right)^{U}$ for a second-order upwind TVD scheme, originally developed by Harten $^{1}$ and later modified and generalized by Yee, ${ }^{7,36}$ are

$$
\left(\phi_{j+1 / 2}^{l}\right)^{U}=1 / 2 \psi\left(a_{j+1 / 2}^{l}\right)\left(g_{j+1}^{l}+g_{j}^{l}\right)-\psi\left(a_{j+1 / 2}^{l}+\gamma_{j+1 / 2}^{l}\right) \alpha_{j+1 / 2}^{l}
$$

$$
\gamma_{j+1 / 2}^{l}=\frac{1}{2} \psi\left(a_{j+1 / 2}^{l}\right) \begin{cases}\left(g_{j+1}^{l}-g_{j}^{l}\right) / \alpha_{j+1 / 2}^{l} & \alpha_{j+1 / 2}^{l} \neq 0 \\ 0 & \alpha_{j+1 / 2}^{l}=0\end{cases}
$$

Examples of the limiter function $g_{j}^{l}$ can be expressed as

$$
\begin{aligned}
& g_{j}^{l}=\operatorname{minmod}\left(\alpha_{j-1 / 2}^{l}, \alpha_{j+1 / 2}^{l}\right) \\
& g_{j}^{l}=\left\{\alpha_{j-1 / 2}^{l}\left[\left(\alpha_{j+1 / 2}^{l}\right)^{2}+\delta\right]+\alpha_{j+1 / 2}^{l}\left[\left(\alpha_{j-1 / 2}^{l}\right)^{2}+\delta\right]\right\} \\
& /\left[\left(\alpha_{j+1 / 2}^{l}\right)^{2}+\left(\alpha_{j-1 / 2}^{l}\right)^{2}+2 \delta\right] \\
& g_{j}^{l}=S \cdot \max \left[0, \min \left(2\left|\alpha_{j+1 / 2}^{l}\right|, S \cdot \alpha_{j-1 / 2}^{l}\right),\right. \\
& \left.\min \left(\left|\alpha_{j+1 / 2}^{l}\right|, 2 S \cdot \alpha_{j-1 / 2}^{l}\right)\right], \quad S=\operatorname{sgn}\left(\alpha_{j+1 / 2}^{l}\right) \\
& g_{j}^{l}=\operatorname{minmod}\left[2 \alpha_{j-1 / 2}^{l}, 2 \alpha_{j+1 / 2}^{l}, 1 / 2\left(\alpha_{j+1 / 2}^{l}+\alpha_{j-1 / 2}^{l}\right)\right]
\end{aligned}
$$

Here, $\delta$ is a small parameter. In practical calculations, $10^{-7}$ $\leq \delta \leq 10^{-5}$ is a commonly used range. The last limiter, Eq. (23f), is due to Woodward and Colella. ${ }^{40}$

\section{MUSCL Approach}

The numerical flux function $\tilde{\boldsymbol{F}}_{j \pm 1 / 2, k}$ for a MUSCL-type approach, together with Roe's type of approximate Riemann solver, for an upwind scheme as described in Yee ${ }^{26,41}$ can be expressed as

$$
\tilde{\boldsymbol{F}}_{j+1 / 2, k}=1 / 2\left[\boldsymbol{F}\left(\boldsymbol{U}_{j+1 / 2}^{R}\right)+\boldsymbol{F}\left(\boldsymbol{U}_{j+1 / 2}^{L}\right)-\hat{R}_{j+1 / 2} \hat{\Phi}_{j+1 / 2}\right]
$$

The values $\boldsymbol{F}\left(\boldsymbol{U}_{j+1 / 2}^{R}\right)$ and $\boldsymbol{F}\left(\boldsymbol{U}_{j+1 / 2}^{L}\right)$ are the flux function $F$ evaluated at $U_{j+1 / 2}^{R}$ and $U_{j+1 / 2}^{L}$, respectively, with

$$
\begin{gathered}
\boldsymbol{U}_{j+1 / 2}^{R}=\boldsymbol{U}_{j+1, k}-1 / 4\left[(1-\bar{\eta}) \widetilde{\Delta_{j+3 / 2}}+(1+\eta) \widetilde{\Delta_{j+1 / 2}}\right] \\
\boldsymbol{U}_{j+1 / 2}^{L}=\boldsymbol{U}_{j, k}+1 / 4\left[(1-\bar{\eta}) \widetilde{\Delta_{j-1 / 2}}+(1+\eta) \widetilde{\Delta_{j+1 / 2}}\right]
\end{gathered}
$$

where $\bar{\eta}$ is a parameter to control the spatial accuracy of the scheme to be discussed shortly. The limiters $\Delta_{j+1 / 2}$ and $\Delta_{j} \widetilde{F}^{\prime}$ 
can be

$$
\begin{aligned}
& {\widetilde{\Delta_{j+1 / 2}}}=\operatorname{minmod}\left(\Delta_{j+1 / 2}, \beta \Delta_{j-1 / 2}\right) \\
& {\widetilde{\Delta_{j+1 / 2}}}^{\approx}=\operatorname{minmod}\left(\Delta_{j+1 / 2}, \beta \Delta_{j+3 / 2}\right)
\end{aligned}
$$

with

$$
\operatorname{minmod}(x, \beta y)=\operatorname{sgn}(x) \cdot \max \{0, \min [|x|, \beta y \operatorname{sgn}(x)]\}
$$

and $\beta=(3-\bar{\eta}) /(1-\bar{\eta})$, where $\Delta_{j+1 / 2}=\boldsymbol{U}_{j+1, k}-\boldsymbol{U}_{j, k}$. For $\bar{\eta}=-1$, $\Delta_{j+1 / 2}$ and $\Delta_{j} \widetilde{\mp}_{1 / 2}$ can be the same limiter as Eqs. (23) except the arguments are now $\Delta_{j \pm 1 / 2}$ instead of $\boldsymbol{\alpha}_{j \pm 1 / 2}$.

The vector $\hat{\boldsymbol{R}}_{j+1 / 2}$ is the eigenvector of $\boldsymbol{A}$ evaluated at some symmetric average of $\boldsymbol{U}_{j+1 / 2}^{R}$ and $\boldsymbol{U}_{j+1 / 2}^{L}$; i.e.,

$$
\hat{\boldsymbol{R}}_{j+1 / 2}=\boldsymbol{R}_{x}\left(\boldsymbol{U}_{j+1 / 2}^{R}, \boldsymbol{U}_{j+1 / 2}^{L}\right)
$$

and the elements of $\hat{\boldsymbol{\Phi}}_{j+1 / 2}$ are

$$
\hat{\phi}_{j+1 / 2}^{l}=\psi\left(\hat{a}_{j+1 / 2}^{l}\right) \hat{\alpha}_{j+1 / 2}^{l}
$$

where again $\hat{a}_{j+1 / 2}^{l}$ and $\hat{\alpha}_{j+1 / 2}^{l}$ are evaluated at some symmetric average of $U_{j+1 / 2}^{R}$ and $U_{j+1 / 2}^{L}$ and

$$
\begin{gathered}
\hat{a}_{j+1 / 2}^{l}=a_{x}^{l}\left(\boldsymbol{U}_{j+1 / 2}^{R}, \boldsymbol{U}_{j+1 / 2}^{L}\right) \\
\hat{\alpha}_{j+1 / 2}=\hat{\boldsymbol{R}}_{j+1 / 2}^{-1}\left(\boldsymbol{U}_{j+1 / 2}^{R}, \boldsymbol{U}_{j+1 / 2}^{L}\right)
\end{gathered}
$$

Here, the spatial order of accuracy pertaining to the scheme with the limiter not present [i.e., remove the tildes from Eqs. $(24 \mathrm{~b}, \mathrm{c})]$ is determined by the value of $\bar{\eta}$. For example, if $\bar{\eta}=-1$, the scheme is fully upwind, and if $\bar{\eta}=0$, it is Fromm's scheme. When $\bar{\eta}=1 / 3$, the scheme is third-order, and when $\bar{\eta}=1$, it is the regular three-point central difference scheme.

The operations count between Eqs. (21-23) and Eq. (24) is within $30 \%$ for a perfect gas. However, due to extra evaluation in the curve fitting between the left and right states in an equilibrium real gas for Eqs. (24), additional computation is required for the MUSCL approach. The slight advantage of Eqs. (24) over Eqs. (21-23) is that Eqs. (24) can be spatially third-order accurate. One word of caution is that experiences with the third-order case $(\bar{\eta}=1 / 3)$ do not show a very visible improvement over the second-order case for the problem with discontinuities. Part of the reason is that all TVD-type schemes reduce to first-order at points of extrema regardless of the order of accuracy at smooth regions.

\section{Conservative Linearized Form for Steady-State Applications}

To solve for $U^{n+1}$ in Eq. (21), one normally needs to solve a set of nonlinear algebraic equations iteratively. One way to avoid this is to linearize the implicit operator and solve the linearized form by other means. If we follow the same procedure as in Refs. 8 and 36, a conservative linearized form of Eqs. (21) can be written as

$$
\begin{aligned}
\{I+ & \theta\left[\frac{\Delta t}{\Delta x}\left(\boldsymbol{H}_{j+1 / 2, k}^{x}-\boldsymbol{H}_{j-1 / 2, k}^{x}\right)^{n}\right. \\
& \left.\left.+\frac{\Delta t}{\Delta y}\left(\boldsymbol{H}_{j, k+1 / 2}^{y}-\boldsymbol{H}_{j, k-1 / 2}^{y}\right)^{n}-\Delta t \overline{\boldsymbol{D}}_{j, k}^{n}\right]\right\} \boldsymbol{E}^{n} \\
= & -\frac{\Delta t}{\Delta x}\left(\tilde{\boldsymbol{F}}_{j+1 / 2, k}^{n}-\tilde{\boldsymbol{F}}_{j-1 / 2, k}^{n}\right) \\
& \left.-\frac{\Delta t}{\Delta y}\left(\tilde{\boldsymbol{G}}_{j, k+1 / 2}^{n}-\tilde{\boldsymbol{G}}_{j, k-1 / 2}^{n}\right)+\Delta t \boldsymbol{S}_{j, k}^{n}\right]
\end{aligned}
$$

$$
\boldsymbol{E}^{n}=\boldsymbol{U}^{n+1}-\boldsymbol{U}^{n}
$$

where

$$
\begin{gathered}
\boldsymbol{H}_{j+1 / 2, k}^{x}=1 / 2\left(\boldsymbol{A}_{j+1, k}-\boldsymbol{\Omega}_{j+1 / 2, k}^{x}\right) \\
\boldsymbol{H}_{j, k+1 / 2}^{y}=1 / 2\left(\boldsymbol{B}_{j, k+1}-\boldsymbol{\Omega}_{j, k+1 / 2}^{y}\right)
\end{gathered}
$$

Note that the matrices $\boldsymbol{H}_{j+1 / 2, k}^{x}$ and $\boldsymbol{H}_{j+1 / 2, k}^{y}$ in Eqs. (25) are different from the variable $H$ in Eq. (9). The nonstandard notation

$$
\boldsymbol{H}_{j+1 / 2, k}^{x} \boldsymbol{E}=1 / 2\left(\boldsymbol{A}_{j+1, k} \boldsymbol{E}_{j+1, k}-\boldsymbol{\Omega}_{j+1 / 2, k}^{x} \boldsymbol{E}\right)^{n}
$$

is used and

$$
\begin{aligned}
& \boldsymbol{\Omega}_{j+1 / 2, k}^{x} \boldsymbol{E}=\boldsymbol{R}_{j+1 / 2} \operatorname{diag}\left[\psi\left(a_{j+1 / 2}^{l}\right)\right] \boldsymbol{R}_{j+1 / 2}^{-1}\left(\boldsymbol{E}_{j+1, k}-\boldsymbol{E}_{j, k}\right) \\
& \boldsymbol{\Omega}_{j, k+1 / 2}^{y} \boldsymbol{E}=\boldsymbol{R}_{k+1 / 2} \operatorname{diag}\left[\psi\left(a_{k+1 / 2}^{l}\right)\right] \boldsymbol{R}_{k+1 / 2}^{-1}\left(\boldsymbol{E}_{j, k+1}-\boldsymbol{E}_{j, k}\right)
\end{aligned}
$$

Here, $\boldsymbol{A}_{j+1, k}, \boldsymbol{B}_{j . k+1}$ are Jacobians of $\boldsymbol{F}$ and $\boldsymbol{G}$ evaluated at $(j+1, k)$ and $(j, k+1)$. The value $\bar{D}_{j, k}$ is the Jacobian of the source term $\boldsymbol{S}$ evaluated at $(j, k)$, and $\boldsymbol{E}_{j, k}=\boldsymbol{U}_{j, k}^{n+1}-\boldsymbol{U}_{j, k}^{n}$. The expression $\operatorname{diag}\left(z^{l}\right)$ denotes a diagonal matrix with diagonal elements $z^{\prime}$. This conservative linearized implicit operator as well as the nonconservative linearized implicit operator ${ }^{8,36}$ was renamed two years later by Barth $^{42}$ as the approximate Jacobian linearization. To compute Eqs. (25f) and (25g), a triple matrix multiplication of dimension $(n s+3, n s+3)$ has to be performed at every grid point. For steady-state applications, one can simplify Eqs. (25f) and (25g) as

$$
\begin{aligned}
& \boldsymbol{\Omega}_{j+1 / 2, k}^{x} \boldsymbol{E}=\mathfrak{T}_{x} I\left(\boldsymbol{E}_{j+1, k}-\boldsymbol{E}_{j, k}\right) \\
& \boldsymbol{\Omega}_{j, k+1 / 2}^{y} \boldsymbol{E}=\mathfrak{T}_{y} I\left(\boldsymbol{E}_{j, k+1}-\boldsymbol{E}_{j, k}\right)
\end{aligned}
$$

The scalar values $\mathfrak{N}_{x}$ and $\mathfrak{T C}_{y}$ are

$$
\begin{aligned}
& \mathfrak{N}_{x}=\max _{l} \psi\left(a_{j+1 / 2}^{l}\right) \\
& \mathfrak{M}_{y}=\max _{l} \psi\left(a_{k+1 / 2}^{l}\right)
\end{aligned}
$$

and $I$ is the identity matrix. Note that Eqs. (25h) and (25i) involve scalar multiplications only. The solution using Eqs. (25) is still second-order (or third-order) accurate after it reaches steady-state. Other linearized treatments can be found in Refs. 8 and 36.

Here, we would like to point out an important distinction between the flux-vector splitting ${ }^{43,44}$ and the local characteristic approach for implicit methods. Unlike flux-vector splitting approaches, ${ }^{43,44}$ implicit methods, Eqs. (25), employing the local characteristic approach do not require the Jacobian of the $\boldsymbol{F}^{ \pm}$fluxes. ${ }^{2,26}$ Here, $\boldsymbol{F}^{ \pm}$is the portion of the flux with positive/negative eigenvalues. In many instances, the Jacobian of $F^{ \pm}$is relatively difficult and expensive to obtain.

\section{Stiff Source Terms, ADI Approaches, and Relaxation Methods}

The stiff terms $\bar{D}_{j, k}^{n}$ of the implicit operator, Eqs. (25), complicate the solution procedures for the commonly used ADI procedures. Normally, if $\overline{\boldsymbol{D}}_{j, k}^{n}$ are not stiff, one can reformulate Eqs. (25) by an ADI procedure like the Beam and Warming ${ }^{45}$ algorithm for an efficient solution process. Unfortunately, the $\overline{\boldsymbol{D}}_{j, k}^{n}$ considered here are stiff; consequently, the additional higher-order terms due to the ADI formulation no longer can be ignored. In a different context, Van Dalsem and Steger ${ }^{46}$ suggested a remedy if $\overline{\boldsymbol{D}}_{j, k}^{n}$ is a diagonal matrix with identical diagonal elements, However, for chemically reacting flows, the matrix $\bar{D}_{j, k}^{n}$ is full for the upper $(n s, n s+3)$ entities and no straightforward efficient way of using ADI approaches for nonlinear system cases with general stiff source terms can be found.

The straightforward way of solving Eqs. (21) as a set of nonlinear systems of equations iteratively or solving the 
linearized form Eqs. (25) or similar forms in a non-ADI appears to be quite expensive for large systems. Recently, Gnoffo, McCandless, and Yee ${ }^{11}$ successfully demonstrated the usefulness of a point-relaxation method on the implicit symmetric TVD scheme of Eqs. (25) for a loosely coupled chemically nonequilibrium flow computations. Here, a similar point-relaxation or line-relaxation method is proposed for the fully coupled system of Eqs. (18). Despite the fact that a larger equation set is involved than in Ref. 11, the extra operations are minimized by making use of the simplification procedure of Sec. II. For a point-relaxation method, the size of the matrix inversion for Eqs. $(25)$ is $(n s+3, n s+3)$ as opposed to the loosely coupled method of Ref. 11, where the size is $(n s, n s)$. The gain in the freedom of controlling the appropriate amount of numerical dissipation in each individual wave more than compensates for the extra expense. More important, solving the fully coupled system is believed to have a better convergence rate than the loosely coupled approach. All of the necessary terms required for the implicit scheme of Eqs. (25) are derived in Sec. II. The implicit operator of Eq. (25a) is diagonally dominant for $\bar{D}_{j, k}=0$. Therefore, one has to make sure that the source term does not destroy the diagonally dominant property that is required for some relaxation methods. Preliminary results using the fully coupled fully implicit symmetric TVD relaxation algorithm of Eqs. (21) by Gnoffo ${ }^{29}$ show a drastic improvement in the convergence rate over the loosely coupled method of Gnoffo and McCandless. ${ }^{19}$ In the following subsection, an even simpler form than that of Eqs. (25) is proposed.

\section{Implicit Algorithm with Explicit Coupling Between Fluid and Species Equations}

To avoid the inversion of large matrices, one can further simplify Eqs. (25) as suggested by Yee ${ }^{25,26}$ by requiring that the coupling between the fluid and species equations be explicit. With this relaxed requirement, one effectively solves the fluid and species equations separately. Unlike the loosely coupled method used in Ref. 11 or the chemistry-split technique used in Ref. 47, the eigenvalues and eigenvectors of the fully coupled equations are coupled explicitly between the fluid and species equations. This can be accomplished by partitioning the $U, F, G, S, \bar{D}$ in the same way as in Eqs. (19) and partitioning the Jacobians $\boldsymbol{A}$ and $\boldsymbol{B}$ and the numerical fluxes $\widetilde{\boldsymbol{F}}_{j \pm 1 / 2, k}$ and $\widetilde{\boldsymbol{G}}_{j, k \pm 1 / 2}$ similarly. First one solves the fluid equations implicitly and then uses the result $\left(U^{I I}\right)^{n+1}$ to solve the species equation implicitly. See $\mathrm{Yee}^{26}$ for a discussion.

With this simplified procedure, one only has to solve two reduced systems of dimensions 3 and $n s$. For line-relaxation methods and $n s>3$, this procedure can provide a large savings in operations count. Although numerical experiments on this simplified procedure have not been done yet, we expect the current method will give a faster convergence rate than the method of Gnoffo et al. ${ }^{11}$ in which they demonstrated that their method is applicable to many three-dimensional bluntbody problems. If point relaxation were used, the only difference between the two methods is that Ref. 11 uses the eigenvalues and eigenvectors of the individual subsystems (fluid and species) alone, whereas the curent method uses the eigenvalues and eigenvectors of the full system. The use of the full eigenvalues' and eigenvectors' set for the current method is believed to enhance the coupling between the two systems without imposing additional conditions as is the case in Ref. 11.

For steady-state application, an algorithm using the TVD scheme for the viscous flows is to difference the hyperbolic terms the same way as before and then central-difference the viscous terms. The final algorithm is the same as Eqs. (25), except that the spatial central-differencing of the viscous term is added to the right-hand side of Eqs. (25). Numerical tests, comparison with other approaches, and recommendations will be reported in a future publication.

\section{Concluding Remarks}

Two numerical algorithms are proposed for hyperboli conservation laws that are suitable for large systems of thermally and chemically nonequilibrium flows in the hyper. sonic regime. The specific properties of the governing equa tions for fluid flow of this type were taken into consideration for more efficient solution procedures. The main areas of consideration are to minimize operation counts, increase th allowable time-step constraint imposed by the stiff sourc terms, and expand the shock-capturing capability beyond classical approaches. Details of all the considerations are described. A preliminary test problem shows certain advan tages of the proposed semi-implicit high-resolution shock-capturing scheme over the classical ways of supplying numerica dissipation. More numerical testing and study will be pursued in the immediate future.

\section{References}

${ }^{1}$ Harten, A., "On a Class of High Resolution Total-Variation Stable Finite-Difference Schemes," SIAM Journal of Numerical Analysis, Vol. 21, No. 1, Feb. 1984, pp. 1-23.

${ }^{2}$ Yee, H. C., "Construction of Explicit and Implicit Symmetric TVD Schemes and Their Applications," Journal of Computational Physics, Vol. 68, Jan. 1987, pp. 151-179; also, NASA TM-86775 July 1985 .

${ }^{3}$ Roe, P. L., "Some Contributions to the Modelling of Discontinu ous Flows," Lectures in Applied Mathematics, Vol. 22, American Mathematical Society, Providence, RI, 1985, pp. 163-194.

${ }^{4}$ Osher, S. and Chakravarthy, S., "Very High Order Accurate TVD Schemes," The IMA Volumes in Mathematics and Its Applications, Vol. 2, Springer-Verlag, New York, 1986, pp. 229-274.

${ }^{5}$ Jameson, A. and Lax, P. D., "Conditions for the Construction of Multi-Point Total Variation Diminishing Difference Schemes," Inst. for Computer Applications in Science and Engineering, Hampton VA, Rept. 86-18, March 1986, pp. 361-382.

"Yee, H. C., "On Symmetric and Upwind TVD Schemes," Proceedings of the 6th GAMM Conf. on Numerical Methods in Fluid Mechanics, Friedr. Vieweg \& Sohn, Braunschweig/Wiesbaden, FRG 1986, pp. 399-403.

${ }^{7}$ Yee, H. C., "Numerical Experiments with a Symmetric High-Res olution Shock-Capturing Scheme," NASA TM-88325, June 1986.

${ }^{8}$ Yee, H. C. and Harten, A., "Implicit TVD Schemes for Hyper bolic Conservation Laws in Curvilinear Coordinates," AIAA Jour nal, Vol. 25, Feb. 1987, pp. 266-274.

${ }^{9}$ Moon, Y. J. and Yee, H. C., "Numerical Simulation by TVD Schemes of Complex Shock Reflections from Airfoils at High Angle of Attack," AIAA Paper 87-0350, Jan. 1987.

${ }^{10}$ Carofano, G. C., "Blast Computation Using Harten's Total Variation Diminishing Scheme," U.S. Army Armament \& Research and Development Center, Watervliet, NY, ARLCB-TR-84029, Oct. 1984.

${ }^{11}$ Gnoffo, P. A., McCandless, R. S., and Yee, H. C., "Enhance ments to Program LAURA for Efficient Computation of Three Dimensional Hypersonic Flow," AIAA Paper 87-0280, Jan. 1987.

${ }^{12}$ Roe, P. L., "Generalized Formulation of TVD Lax-Wendrof Schemes," Inst. for Computer Applications in Science and Engineer ing, Hampton, VA, Rept. 84-53, Oct. 1984.

${ }^{13}$ Davis, S. F., "TVD Finite Difference Schemes and Artificia Viscosity," Inst. for Computer Applications in Science and Engineer ing, Hampton, VA, Rept. 84-20, June 1984.

${ }^{14}$ Gear, C. W., Numerical Initial Value Problems in Ordinar Differential Equations, Prentice-Hall, Englewood Cliffs, NJ, 1971

${ }^{15}$ Kreiss, H.-O., "Problems with Different Time Scales for PDE, Communication in Pure and Applied Mathematics, Vol. 32, 1980, pp 399-439.

${ }^{16}$ Guerra, J. and Gustafsson, B., "A Semi-Implicit Method fo Hyperbolic Problems with Different Time Scales," SIAM Journal Numerical Analysis, 1986 (to be published).

${ }^{17} \mathrm{Kee}, \mathrm{R}$. J. and Miller, J. A., "Computational Modeling of Flam Structure," Physica D, Nonlinear Phenomena, Vol. 12D, Nos. $1^{-3}$ July 1984, pp. 198-211.

${ }^{18}$ Bussing, T. R. A. and Murman, E. M., "Finite Volume Metho for the Calculation of Compressible Chemically Reacting Flows, AIAA Paper 85-0331, Jan. 1985.

${ }^{19}$ Gnoffo, P. A. and McCandless, R. S., "Three-Dimension" AOTV Flowfields in Chemical Nonequilibrium," AIAA Pap 86-0230, Jan. 1986. 
${ }^{20}$ Drummond, J. P., Hussaini, M. Y., and Zang, T. A., "Spectral Methods for Modeling Supersonic Chemically Reacting Flow Fields," AIAA Paper 85-0302, Jan. 1985.

${ }^{21} \mathrm{Li}, \mathrm{C}$. P., "Implicit Method for Computing Chemically Reacting Flow," NASA TM-58274, Sept. 1986.

${ }^{22}$ Eklund, D. R., Hassan, H. A., and Drummond, J. P., "The Efficient Calculation of Chemically Reacting Flow," AIAA Paper 86-0563, Jan. 1986.

${ }^{23}$ Uenishi, K. and Rogers, R. C., "'Three-Dimensional Computation of Transverse Hydrogen Jet Combustion in a Supersonic Air Stream," AIAA Paper 87-0089, Jan. 1987.

${ }^{24}$ Eberhardt, S. and Brown, K., "A Shock Capturing Technique for Hypersonic, Chemically Relaxing Flows," AIAA Paper 86-0231, Jan. 1986.

${ }^{25}$ Yee, H. C., "Numerical Aspects in Computing Hyperbolic Conservation Laws with Stiff Source Terms," SIAM Conf. on Numerical Combustion, San Francisco, CA, March 9-11, 1987.

${ }^{26} Y e e$, H. C., "Upwind and Symmetric Shock-Capturing Schemes," NASA TM-89464, May 1987, updated version: "A Class of High-Resolution Explicit and Implicit Shock-Capturing Methods," von Kármán Institute Lecture Notes in Computation Fluid Dynamics, March 1989.

${ }^{27} \mathrm{LeV}$ eque, R. J. and Yee, H. C., "A Study of Numerical Methods for Hyperbolic Conservation Laws with Stiff Source Terms," NASA TM-100075, March 1988.

${ }^{28}$ Shinn, J. L., Yee, H. C., and Uenishi, K., "Extension of a Semi-Implicit Shock-Capturing Algorithm for 3-D Fully Coupled Chemically Reacting Flows in Generalized Coordinates," AIAA Paper 87-1577, June 1987.

${ }^{29}$ Gnoffo, P. A., "Governing Equations and Physical Models for Hypersonic Airflows in Thermal and Chemical Nonequilibrium," NASA-TP, 1988 (to be published).

${ }^{30}$ Huang, L. C., "Pseudo-Unsteady Difference Schemes for Discontinuous Solutions of Steady-State, One-Dimensional Fluid Dynamics Problems," Journal of Computational Physics, Vol. 42, 1981, pp. 195-211.

${ }^{31}$ Roe, P. L., “Approximate Riemann Solvers, Parameter Vectors, and Difference Schemes," Journal of Computational Physics, Vol. 43, 1981, pp. 357-372.

${ }^{32}$ Montagné, J.-L., Yee, H. C., Klopfer, G. H., and Vinokur, M., "Hypersonic Blunt Body Computations Including Real Gas Effects,", Proc. 2nd Inter. Conf. on Hyperbolic Problems, Aachen, FRG, March 14-18, 1988.

${ }^{33}$ Yee, H. C., Klopfer, G. H., and Montagné, J.-L., "'High-Resolution Shock-Capturing Schemes for Inviscid and Viscous Hypersonic Flows," NASA-TM, April 1988.
${ }^{34}$ Kwong, C. M., "Numerical Experiments with a Total Variation Diminishing (TVD) MacCormack Scheme," Proceedings of the 6th GAMM Conf. on Numerical Methods in Fluid Mechanics, Friedr. Vieweg \& Sohn, Braunschweig/Wiesbaden, FRG, 1986, pp. 217-224.

${ }^{35}$ Warming, R. F., Kutler, P., and Lomax, H., "Second- and Third-Order Noncentered Difference Schemes for Nonlinear Hyperbolic Equations," AIAA Journal, Vol. 10, Feb. 1973, pp. 189-196.

${ }^{36}$ Yee, H. C., "Linearized Form of Implicit TVD Schemes for the Multidimensional Euler and Navier-Stokes Equations," Computers and Mathematics with Applications, Vol. 12A, No. 4/5, 1986, pp. 413-432.

${ }^{37}$ Pulliam, T. H. and Steger, J. L., "Recent Improvements in Efficiency, Accuracy and Convergence for Implicit Approximate Factorization Algorithms," AIAA Paper 85-0360, Jan. 1985.

${ }^{38}$ Jameson, A., Schmidt, W., and Turkel, E., "Numerical Solutions of the Euler Equations by Finite Volume Methods Using Runge-Kutta Time-Stepping Schemes," AIAA Paper 81-1259, June 1981.

${ }^{39}$ van Leer, B., "Towards the Ultimate Conservation Difference Scheme V, A Second-Order Sequel to Godunov's Method,"' Journal of Computational Physics, Vol. 32, 1979, pp. 101-136.

${ }^{40}$ Woodward, P. and Colella, P., "The Numerical Simulation of Two-Dimensional Fluid Flows with Strong Shocks," Journal of Computational Physics, Vol. 54, 1984, pp. 115-173.

${ }^{41}$ Yee, H. C., "On the Implementation of a Class of Upwind Schemes for System of Hyperbolic Conservation Laws," NASA TM-86839, Sept. 1985.

${ }^{42}$ Barth, T. J., "Analysis of Implicit Local Linearization Techniques for Upwind and TVD Algorithms," AIAA Paper 87-0595, Jan. 1987.

${ }^{43}$ van Leer, B., "Flux-Vector Splitting for the Euler Equations," Institute for Computer Applications in Science and Engineering, Hampton, VA, Rept. 82-30, Sept. 1982.

${ }^{44}$ Steger, J. L. and Warming, R. F., "Flux Vector Splitting of the Inviscid Gasdynamic Equations with Application to Finite Difference Methods," Journal of Computational Physics, Vol. 40, No. 2, 1981 , pp. 263-293.

${ }^{45}$ Beam, R. M. and Warming, R. F., "An Implicit Finite-Difference Algorithm for Hyperbolic Systems in Conservation Law Form," Journal of Computational Physics, Vol. 22, 1976, pp. 87-110.

${ }^{46}$ Van Dalsen, W. R. and Steger, J. L., "Using the Boundary-Layer Equations in Three-Dimensional Viscous Flow Simulation," NASA TM-88241, March 1986

${ }^{47} \mathrm{Li}$, C. P., "Chemistry-Split Techniques for Viscous Reactive Blunt Body Flow Computations," AIAA Paper 87-0282, Jan. 1987.

${ }^{48}$ Vinokur, M., private communication, Sterling Software, Mountain View, CA. 